\title{
Small Scale Structure in Nearby Molecular Gas
}

\author{
Edith Falgarone
}

Laboratoire de Radioastronomie, Ecole Normale Supérieure, 24 rue Lhomond, F-75231 Paris Cedex 05, France

\begin{abstract}
Recent observations at high angular resolution of molecular clouds of low column density have revealed the presence of a conspicuous net of small scale filamentary structures, visible in the ${ }^{12} \mathrm{CO}$ rotational lines only. In addition, the existence of unresolved structure at scales as small as $\sim 200 \mathrm{AU}$ in space and/or velocity space is inferred from the spectral properties of the ${ }^{12} \mathrm{CO}$ and ${ }^{13} \mathrm{CO}$ emission. The resolved structures are part of the hierarchy of structures observed in molecular gas in the Solar Neighborhood and appear as non self-gravitating elements confined by an ambient pressure $P_{0} / k_{B} \sim 3 \times 10^{4} \mathrm{~cm}^{-3} \mathrm{~K}$. We show why these structures might have their origin in the intermittent structures of turbulence in which viscous dissipation is concentrated in space and time.
\end{abstract}

\section{A Short Overview of Past Evidence for Small Scale Structure in Molecular Clouds}

As soon as, in the mid 70's, the first maps of interstellar clouds in the rotational lines of ${ }^{12} \mathrm{CO}$ appeared, it was inferred from the line excitation conditions and radiative transfer (Penzias et al., 1973) that lines were emitted by gas colder than $\sim 10 \mathrm{~K}$ and as dense as $10^{3}$ to $10^{4} \mathrm{~cm}^{-3}$. The ${ }^{12} \mathrm{CO}$ linewidths, $\Delta v \sim$ a few $\mathrm{km} \mathrm{s}^{-1}$, were strikingly non-thermal since the thermal velocity of $\mathrm{CO}$ at $\mathrm{T}=10 \mathrm{~K}$ is $0.05 \mathrm{~km} \mathrm{~s}^{-1}$.

Soon after, it was recognized that such large densities had to fill only less than one hundredth of the volume of molecular complexes because the gas column densities combined with the sizes indicated average gas densities not larger than $\sim 100 \mathrm{~cm}^{-3}$ within clouds of a few pc in size. Yet, lower average densities $\sim 10 \mathrm{~cm}^{-3}$ were derived for giant molecular complexes of size $\sim 50$ pc, (Pérault et al. 1985, Blitz, 1987). This was the starting point of the concept of clumpy molecular clouds. The clumps were then modelled as self-gravitating polytropes of about $\sim 1 \mathrm{pc}$, bounded by the external pressure of a warm interclump medium of density a few tenth $\mathrm{cm}^{-3}$ and kinetic temperature of the order of $8000 \mathrm{~K}$ (Falgarone \& Puget, 1985). In this model, the density fall-off at the clump boundaries was generated by the thermal instability of the interstellar gas (Field, 1965). The interclump medium was heated by the drift of magnetic field lines attached to the clumps in their virialized motions in the gravitational potential well of the complex (Falgarone \& Puget, 1986). 
At about the same epoch, the scale free properties of the hierarchy of structures of molecular clouds seen in the $\mathrm{CO}$ lines started to be unveiled (Larson, 1981), and scaling laws were built between the mass and size of structures, their internal velocity and size. Larson postulated that the scaling laws were reminiscent of the Kolmogorov law which characterizes the power spectrum of incompressible turbulence and suggested that they reflected the turbulent nature of the flows within molecular clouds. On the other hand, Chièze (1987) noticed that the hierarchy of observed structures coincided with the ensemble of the most massive isothermal polytropes (at different temperatures) which are gravitationally stable in an ambient pressure $P_{0}$.

Up to the mid 80's, a description was emerging, in which the structures of molecular clouds at all levels between $\sim 50 \mathrm{pc}$ down to $\sim 1 \mathrm{pc}$ were selfgravitating entities. The nature of the internal energy density was known to be dominantly non-thermal which demanded some refinement of Chièze's description. But the predictions were remarkably in agreement with the observations.

What have we learnt since then? One important step forward has been the discovery that the hierarchy extends down to scales two orders of magnitude smaller, $\sim 0.01 \mathrm{pc}$, at which structures are no longer self-gravitating.

\section{Recent Results from the first IRAM Key-Project}

These recent progresses are examplified by the results obtained in the framework of the first IRAM key-project (Frisch 1997). The goal of this project was to map the environment of low mass dense cores at high angular resolution in the perspective of understanding some of the processes at work in the dissipation of the non-thermal support of molecular clouds. The selected targets are pieces of ordinary gas containing a low mass core with internal velocity dispersion close to thermal. Except for the dense cores themselves which have total $\mathrm{H}_{2}$ column density $N_{\mathrm{H}_{2}} \sim$ a few $10^{22} \mathrm{~cm}^{-2}$, the average column density of the fields mapped is quite low, $N_{\mathrm{H}_{2}} \sim$ a few $10^{20} \mathrm{~cm}^{-2}$ at the parsec scale.

\subsection{Resolved Structure}

Figure 1 displays the maps of ${ }^{12} \mathrm{CO}(2-1),{ }^{13} \mathrm{CO}(2-1)$ and $\mathrm{C}^{18} \mathrm{O}(1-0)$ line integrated emission in one of the fields studied, a high latitude cloud in the vicinity of the Polaris star. The pattern seen in the ${ }^{12} \mathrm{CO}$ line is quite different from that seen in the ${ }^{13} \mathrm{CO}$ and $\mathrm{C}^{18} \mathrm{O}$ lines. In ${ }^{12} \mathrm{CO}$, there is almost no structure visible except for a few barely resolved hot spots while in the ${ }^{13} \mathrm{CO}$ and $\mathrm{C}^{18} \mathrm{O}$ lines an arc like structure which delineates high column density gas (the dense core) is clearly visible.

Figure 2 shows the large amount of small scale structure present in the ${ }^{12} \mathrm{CO}(2-1)$ maps as soon as the different velocities are separated from one 

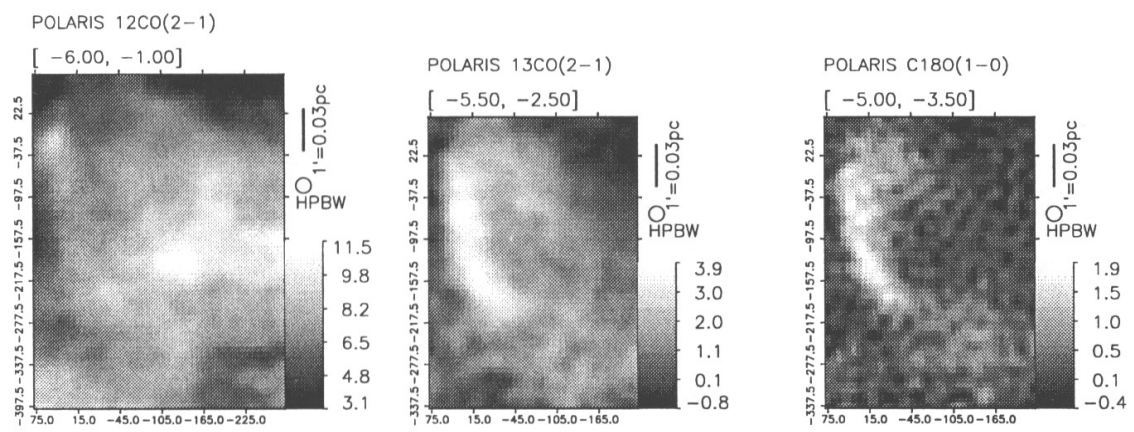

Fig. 1. Maps of line integrated area in the Polaris field. The different transitions are indicated above each panel. The linear scale is shown, together with the angular resolution (HPBW) and the intensity scale expressed in $\mathrm{K} \mathrm{km} \mathrm{s}^{-1}$. The numbers between brackets give the velocity range of integration, in $\mathrm{km} \mathrm{s}^{-1}$. The coordinates offset are in arcsec.

another. The channel maps of Fig. 2 reveal a net of filamentary or more compact structures, not clearly connected to the arc visible in the rare isotopes maps. Note that some of the filaments have width at half-maximum of the order of the telescope resolution at that frequency, HPBW $=14$ " or $0.007 \mathrm{pc}$ or $1800 \mathrm{AU}$ at the distance of the cloud $(100 \mathrm{pc})$.

The discovery of such a small scale filamentary structure in the ${ }^{12} \mathrm{CO}$ lines was unexpected because the weak ${ }^{12} \mathrm{CO}$ emission observed at low angular resolution in cloud edges has long been thought to originate in a low density homogeneous medium that would subthermally excite the CO rotational lines. It is indeed a beam-diluted emission of bright small scale filaments, identified only in large maps observed at high angular resolution. A similar result has been found by Falgarone \& Phillips (1996). Here, higher J ${ }^{12} \mathrm{CO}$ transitions are used to constrain the parameters of the gas emitting in a small filament detected in a cloud edge. Solutions range from dense $n_{\mathrm{H}_{2}}=10^{3} \mathrm{~cm}^{-3}$ gas at $T_{k} \sim 25 \mathrm{~K}$ to lower density gas $n_{\mathrm{H}_{2}} \sim 200 \mathrm{~cm}^{-3}$ at $T_{k} \sim 250 \mathrm{~K}$, opening the possibility that, unlike cold gas in dense cores, these filaments seen in ${ }^{12} \mathrm{CO}$ are much warmer. These two sets of results also show that it is the gas which emits in the wings of the CO lines and has the broadest velocity coverage which has the highest level of small scale structure in ${ }^{12} \mathrm{CO}$. This component is not visible in ${ }^{13} \mathrm{CO}$, unless huge integration times are used.

\subsection{Sub-beam Structure}

In addition to the resolved small scale structure, the existence of structure at much smaller size scales is inferred from two very well defined properties of the CO line profiles disclosed in the above papers. These properties are illustrated in Figure 3 for the Polaris field. The first property, is the constancy of the 

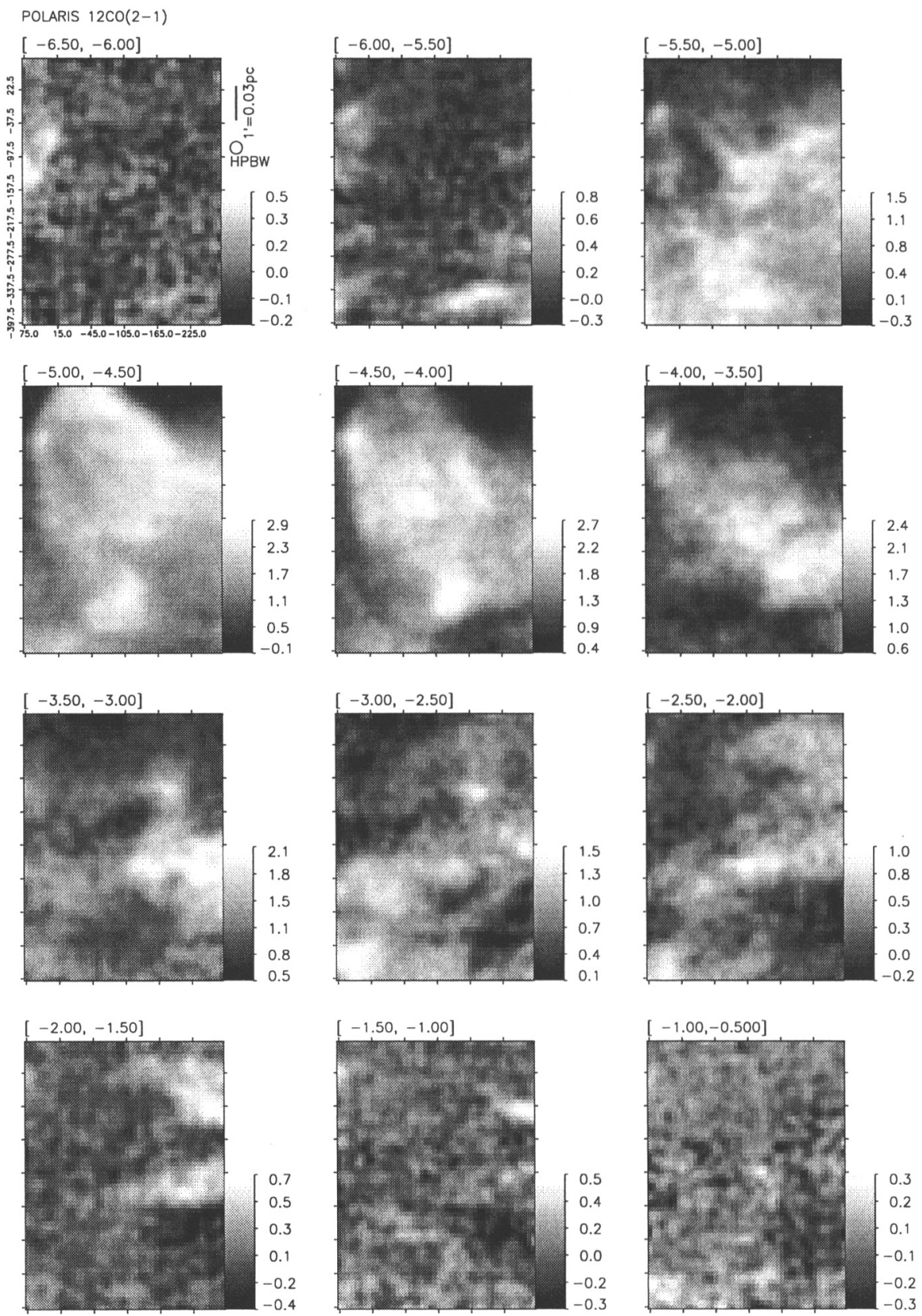

Fig. 2. Space-velocity maps of the ${ }^{12} \mathrm{CO}(2-1)$ emission in the Polaris field. The velocity intervals, in $\mathrm{km} \mathrm{s}^{-1}$, are given at the top of each panel and the grey scale calibration in $\mathrm{K} \mathrm{km} \mathrm{s}^{-1}$, different for each velocity interval to emphasize the existence of the weak filamentary structure, is given at the right side of each map. 
$\mathrm{J}=2-1$ to $\mathrm{J}=1-0$ line ratio $\mathrm{R}(2-1 / 1-0) \sim 0.65$ across the line profiles and for the two ${ }^{12} \mathrm{CO}$ and ${ }^{13} \mathrm{CO}$ isotopic lines. The second property is the smoothness of the line profiles, although the channel width is as small as $0.05 \mathrm{~km} \mathrm{~s}^{-1}$.
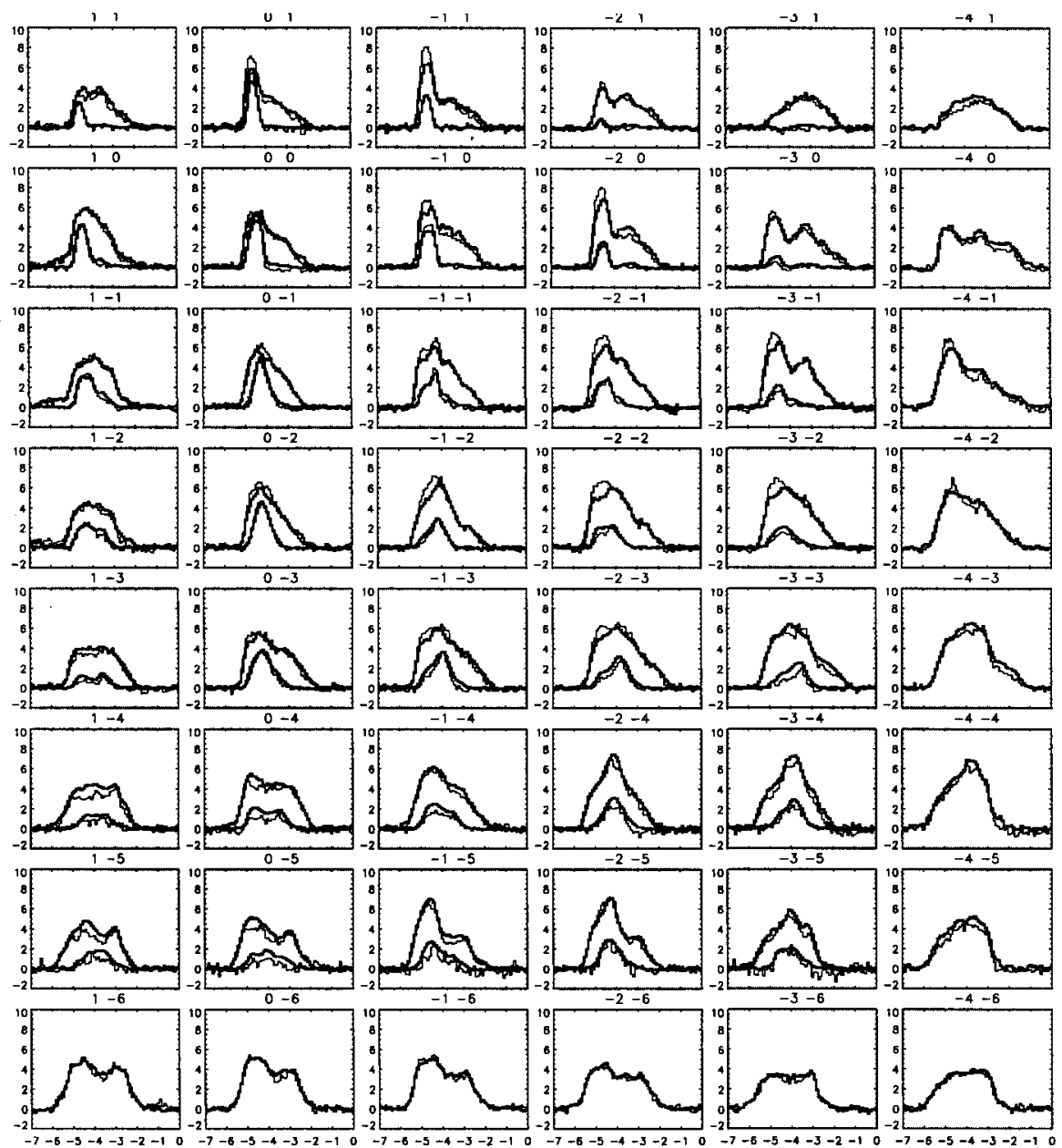

Fig. 3. Maps of ${ }^{12} \mathrm{CO}$ and ${ }^{13} \mathrm{CO}$ integrated spectra over 1' fields in Polaris. The temperature scale is antenna temperature corrected for error beam contributions. Thick lines are for the ${ }^{12} \mathrm{CO}$ and ${ }^{13} \mathrm{CO} \mathrm{J}=1-0$ transitions (same scale). The intensities of the ${ }^{12} \mathrm{CO}(2-1)$ and ${ }^{13} \mathrm{CO}(2-1)$ line profiles (thin lines) have all been divided by the same factor $\mathrm{R}(2-1 / 1-0)=0.65$

From the constancy of the line ratio in space and velocity (i.e. across the line profiles) we derive that the excitation temperature of the rotational lines 
is independent of velocity i.e. there exists very little radiative coupling among regions on the same line of sight at neighboring velocities. These conditions for line formation in which there is little shadowing of the emitting regions in phase-space are called macroturbulent (see Martin, Sanders \& Hills (1984) for more details). To ensure the small radiative coupling at each velocity, line has to form in cells which have a small filling factor both in real space (in the beam) and in velocity space (in a channel). This condition is met as long as the number of cells in the beam and per channel stays below a given threshold. At the opposite, the line smoothness implies a large number of cells per beam and per channel, as shown in Tauber et al. (1991), the temperature fluctuations from one channel to the next being due to the statistical fluctuations of the number of emitting cells in the beam per channel. These two constraints on the number of emitting cells per beam and per channel are antagonistic. Under the assumption that the cells are all identical and statistically independent, these two conditions are met for cells as small as $l \sim 200 \mathrm{AU}$.

We therefore infer the existence of structures one order of magnitude smaller than the actual resolution of our data in the molecular gas rich in ${ }^{12} \mathrm{CO}$ from the spectral properties of the $\mathrm{CO}$ line emission.

\subsection{The Hierarchy of Scales}

A possible key to the understanding of the physics of molecular clouds and the origin of their structure may be that the main parameters of molecular clouds (their mass, size and internal velocity dispersion) are related by power-law relations.

Figure 4 displays the $\mathrm{H}_{2}$ mass versus size of a large ensemble of structures identified in the 4-dimensional space $T_{L}\left(x, y, v_{z}\right)$ of ${ }^{12} \mathrm{CO}$ data mostly (a few values are derived from ${ }^{13} \mathrm{CO}$ or $\mathrm{C}^{18} \mathrm{O}$ lines). $T_{L}$ is the line temperature at velocity $v_{z}$ and beam position $(x, y)$. The masses and sizes are those given by the authors, unless some scaling was necessary to take the different size definitions into account. The structures are identified in molecular surveys of the central parts of the Galaxy (stars: Dame et al. (1986), open triangles: Solomon et al. (1987)) and of the third quadrant (open hexagons: May et al. (1997)), in the Rosette (crosses) and Maddalena (open squares) molecular clouds (Williams et al., 1994), in non-star-forming clouds (solid triangles: Falgarone \& Pérault (1987), solid squares: (Falgarone et al., 1992), tripods: Lemme et al. (1995), asterisks: Herbertz et al. (1991)), in $\rho$ Ophiuchus (solid hexagons: Loren (1989)) and in the Polaris high latitude cloud (data from the IRAM key-project described above, starred triangles: Heithausen et al. (1997)). In addition, the average values obtained for a set of 10 representative dense cores correspond to the large solid squares, Wang et al. (1995). We have added masses and sizes derived from submillimeter continuum observations of the dust for another set of low mass dense cores (solid squares of intermediate size, Ward-Thomson et al. (1994)) and those derived from CS(5-4) 
observations of massive dense cores in Orion (large open squares, Lada et al. (1997)).

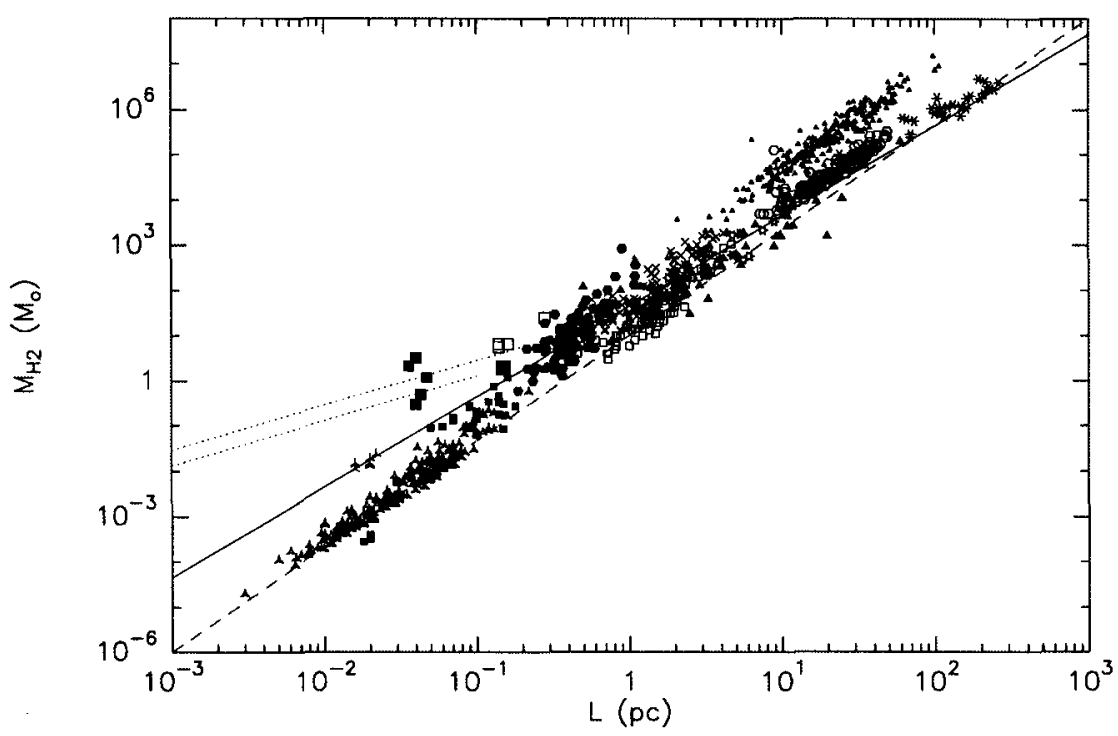

Fig. 4. $\mathrm{H}_{2}$ mass versus size for structures in galactic molecular clouds. The symbols are explained in the text.

In Figure 4, the full line traces the scaling predicted by Chièze (1987):

$$
\frac{M}{L^{2}}=44.5\left(\frac{P_{0} / k_{B}}{3 \times 10^{4} \mathrm{~cm}^{-3} \mathrm{~K}}\right)^{0.5} \mathrm{M}_{\odot} \mathrm{pc}^{-2}
$$

for a pervading pressure $P_{0} / k_{B}=3 \times 10^{4} \mathrm{~cm}^{-3} \mathrm{~K}$. This pressure is the same as that due to the weight of the HI gas layer at mid-plane in the Galaxy (Boulares \& Cox, 1990), also equal to the observed average nonthermal kinetic pressure at small scale in non star forming regions of the Solar Neighnorhood (Falgarone et al., 1992). The two dotted lines in Figure 4 represent the locus of self-gravitating isothermal spheres at $T_{k}=10 \mathrm{~K}$ and $50 \mathrm{~K}$. The dashed line has a slope 2.3, interpreted by Elmegreen \& Falgarone (1996) as a signature of the fractal structure of molecular clouds. It is also the slope found by Heithausen et al. (1997) in the ${ }^{12} \mathrm{CO}$ emission of the high latitude cloud in Polaris, over two orders of magnitude in size.

Many structures lie above the full line, (i) the densest regions of cores in Orion or in nearby clouds, suggesting that these cores, as expected, are not bounded by the ambient pressure of the Solar Neighborhood but by a larger pressure, exerted by their self-gravitating environment, (ii) the structures 
in the molecular ring complexes (Solomon et al. (1987)) suggesting that the ambient pressure there is $\sim 10^{2}$ larger than that in the Solar Neigborhood.

Many structures, especially the smallest, lie below the full line. They have masses much smaller than the marginally stable self-gravitating structures of same size and are therefore in pressure balance with the external pressure. There is indeed little observational evidence that self-gravitating structures have ever been found at scales below that of the dense cores $\sim 0.1 \mathrm{pc}$. If the mass estimates for the smallest scales are correct and the existence of non self-gravitating structures is confirmed, it will prove that a process other than fragmentation driven by gravitational instability is at work in shaping the medium.

\section{Possible Link with the Bursts of Viscous Dissipation of turbulence}

\subsection{The Intermittency of Turbulence in Laboratory Flows and Numerical Simulations}

The velocity field in a turbulent fluid exhibits more spatial structure than a random velocity field in all of the quantities related to increments or derivatives of the velocity field. Vorticity filaments in a turbulent field are more intense and more coherent spatially than in a random velocity field of same power spectrum, She et al. (1990). These vortices are observed in laboratory experiments, (Douady et al., 1991) and are thought to be responsible for the property of intermittency of turbulence, at the origin of the non-Gaussian probability distribution functions (PDFs) of the velocity increments, derivatives and vorticity (Vincent \& Meneguzzi (1991), Porter et al. (1994), Anselmet et al. (1984), Tabeling et al. (1996)). Their diameter ranges between the dissipation scale and the Taylor microscale.

The regions of large vorticity in turbulent flows are associated with those of large dissipation rate of kinetic energy and the two subsets of space where these quantities are concentrated are highly structured and intermittent (Vincent \& Meneguzzi (1991), Porter et al. (1994)). The dissipation of turbulent kinetic energy via the viscous process of elastic collisions between atoms and molecules eventually turns this energy into heat. The rate at which the gas is heated by this process is as large as the intensity of the intermittent event is large, i.e. the local dissipation rate may reach large values for events far in the non-Gaussian wings of the PDFs of velocity increments.

\subsection{The Intermittency of Turbulence in Interstellar Gas}

Experimental results obtained in flow experiments have been scaled to the interstellar medium, under the assumption that the statistical flow properties in turbulence only depend on the kinetic energy transfer rate. In atomic 
clouds, the size of the coherent vortices is estimated to range between $\sim 20$ $\mathrm{AU}$ and $0.03 \mathrm{pc}$. The local heating rate, due to bursts of turbulent viscous dissipation, has been found to exceed all the others heating rates in atomic clouds, by orders of magnitude. The lifetime of the intermittent structures is long enough to heat the gas to temperatures large enough to trigger chemical reactions with activation barriers and endothermic reactions (Falgarone \& Puget,1995). Time-dependent calculations show that, as expected, the abundances of molecules like $\mathrm{OH}, \mathrm{H}_{2} \mathrm{O}, \mathrm{CH}^{+}, \mathrm{HCO}^{+}$, and therefore $\mathrm{CO}$, in these regions can be orders of magnitude larger than in the surrounding colder gas (Falgarone et al., 1995). The recent discovery of unexpected amounts of such molecules in interstellar gas of low extinction might be seen as a signature of intermittent dissipation of turbulence (Lucas \& Liszt, 1996). Taking the effects of magnetic field into account yet reproduces better the observed correlation between $\mathrm{OH}$ and $\mathrm{HCO}^{+}$(Joulain et al., 1997). The intermittency of turbulence creates singularities in interstellar gas. The bursts of viscous dissipation locally heat the gas, triggering the formation of molecular species which in turn increase the radiative cooling of the gas, inducing condensation.

These structures may be too small to be observed individually, although laboratory experiments and simulations show that they are bunched together in space, Porter et al. (1994). Signatures of regions of enhanced vorticity have been searched for in molecular line profiles. Line profiles have been synthesized at different epochs in the evolution of a decaying run of compressible turbulence (Falgarone et al., 1994) and in a random velocity field with a Kolmogorov power spectrum (Dubinski et al., 1995). The results are very comparable except for the existence of very broad and weak line wings in the profiles present only in the turbulent case. These weak line wings are associated to regions of large vorticity within the beam (Lis et al., 1996). Further, the shape of the line profiles is statistically similar to observed line profiles only when the bulk of the turbulent kinetic energy is contained in solenoidal modes, not in compressible modes. This result suggests that the molecular line profiles of interstellar clouds trace vortices rather than shocks.

The above elements do not prove yet that any kind of small scale structure is created in the cold interstellar medium by the intermittent dissipation of turbulent energy. The kind of structures associated with the intermittent viscous dissipation of supersonic turbulence should be observed in all the turbulent components of the interstellar medium. One anticipated signature of this process is the existence of warm filamentary structures temporarily embedded within colder gas.

\section{References}

Anselmet, F., Gagne Y., Hopfinger E.J. \& Antonia R.A. (1984): JFM 140, 63.

Blitz L. (1987): Physical Processes in Interstellar Clouds, eds. Morfill \& Scholer, (Reidel, Dordrecht), 35 
Boulares A. \& Cox D., (1990): ApJ, 365, 544.

Chièze J.-P. (1987): A\&A171 225

Dame T.M., Elmegreen B.G., Cohen R.S. \& Thaddeus P., (1986): ApJ 305, 892.

Douady S., Couder Y. \& Brachet M.E., (1991): Phys. Rev. Letters, 67, 983.

Dubinski J., Narayan R. \& Phillips T.G., (1995): ApJ 448, 226.

Elmegreen B.G. \& Falgarone E. (1996): ApJ 471, 816.

Falgarone E. \& Puget J.-L. (1985): A\&A 142, 157.

Falgarone E. \& Puget J.-L. (1986): A\&A 162, 235.

Falgarone E., \& Pérault M., (1987): Physical Processes in Interstellar Clouds, eds. Morfill \& Scholer, (Reidel, Dordrecht), 59

Falgarone E., Puget J.-L., \& Pérault M. (1992): A\&A, 257, 715

Falgarone E., Lis D.C., Phillips T.G., Pouquet A., Porter D.H. \& Woodward P.R., (1994): ApJ, 436, 728.

Falgarone E. \& Puget J.-L. (1995): A\&A, 293, 840

Falgarone E., Pineau des Forêts G. \& Roueff E., (1995): A\&A, 300, 870.

Falgarone E. \& Phillips T.G. (1996): ApJ 472, 191.

Falgarone E., Panis, J.-F., Heithausen A., Pérault M., Stutzki J., Puget J.-L., \& Bensch F., (1997): A\&A, in press.

Field G.B. (1965): ApJ 142, 531.

Heithausen A., Bensch F., Stutzki J., Falgarone E., Panis J.-F. (1997): A\&A, submitted.

Herbertz R., Ungerechts H. \& Winnewisser G. (1991): A\&A 249, 483.

Lada E.A., Evans N.J. II \& Falgarone E. (1997): ApJ in press.

Joulain K., Falgarone E., Pineau des Forêts G. \& Flower D. (1997) A\&A submitted

Larson R. (1981): MNRAS 194809.

Lemme C., Walmsley C.M., Wilson T.L. \& Muders D. (1995): A\&A, 302, 509.

Lis D.C., Pety J., Phillips T.G. \& Falgarone E. (1996): ApJ 463, 623.

Loren R.B. (1989): ApJ 338, 902.

Lucas R. \& Liszt H. (1996): A\&A, 307, 237

Martin H.M., Sanders D.B. \& Hills R. (1984): MNRAS 20835.

May J., Alvarez H. \& Bronfman L. (1997): A\&A in press.

Penzias A.A., Solomon P.M., Jefferts K.B. \& Wilson R.W. (1973): ApJL 174, L43

Pérault M., Falgarone E., Puget J.-L. (1985):A\&A 152, 371.

Porter D.H., Pouquet A. \& Woodward P.R. (1994): Phys. Fluids, 6, 2133.

She Z.S., Jackson E., Orszag S.A. (1990): Nature, 344, 226.

Solomon P.M., Rivolo A.R., Barrett J. \& Yahil A. (1987) ApJ, 319, 730.

Tabeling P., Zocchi G., Belin F., Maurer J. \& Willaime H. (1996): Phys. Rev. E, 53,1613

Tauber J., Goldsmith P.F. \& Dickman R.L.(1991): ApJ 375, 635.

Vincent A. \& Meneguzzi M., (1991): J. Fluid Mech., 225, 1.

Wang Y., Evans N.J. II, Zhou S. \& Clemens D.P. (1995): ApJ 454, 217.

Ward-Thompson D., Scott P.F., Hills R.E. \& André P. (1994): MNRAS, 268, 276.

Williams J.P., de Geus E.J. \& Blitz L. (1994): ApJ 428, 693. 